\title{
Growth factors and diabetic nephropathy: kidney structure and therapeutic interventions
}

\author{
R .W. B ilous ${ }^{1}$, P. Fioretto ${ }^{2}$, P. Czernichow ${ }^{3}$, K. D rummond ${ }^{4}$ \\ ${ }^{1}$ Department of Medicine, University of Newcastle upon Tyne, UK \\ ${ }^{2}$ Department of Internal Medicine, University of Padova, Italy \\ ${ }^{3}$ Hopital Robert Debre, Paris, France \\ ${ }^{4}$ Montreal Children's Hospital, Montreal, Quebec, Canada
}

The second session of the conference that was allocated to nephropathy concentrated on structural and therapeutic aspects. This review will report the session by discussing several aspects of glomerular structure and function in insulin-dependent diabetes mellitus (IDDM) and also address some aspects of heritability of glomerulopathy. The effect of residual beta-cell function will also be described and the possibility of a glycaemic threshold for nephropathy discussed. Finally, the evidence for effective therapeutic intervention at the various stages of nephropathy will be reviewed.

\section{Renal structural/functional relationships in early IDDM}

It is known that in IDDM the clinical manifestations of nephropathy, proteinuria, declining glomerular filtration rate (GFR) and hypertension, are consequent to the progression to advanced stages of a constellation of pathological lesions including glomerular (GBM) and tubular (TBM) basement membrane thickening, increased mesangial fractional volume $[\mathrm{Vv}(\mathrm{Mes} / \mathrm{glom})]$ and interstitial fractional volume $[\mathrm{Vv}($ Int/cortex $)]$, arteriolar hyalinosis and an increased number of globally sclerotic glomeruli [1]. The majority of studies on structural-functional relationships in IDDM have been cross-sectional, making it difficult to ascribe a central causative role to any

Participants: M. Mauer, University of Minnesota, Minneapolis, USA

M. Steffes, University of Minnesota, Minneapolis, USA

G. Boner, Rabin Medical Center, Petah Tikva, Israel

C. E. Mogensen, Kommunehospitalet, Aarhus, Denmark

Corresponding author: Dr. R. W. Bilous, Diabetes Care Centre, Middlesbrough General Hospital, Middlesbrough TS5 5AZ, UK single lesion. Nevertheless, among these structural changes, $\mathrm{Vv}(\mathrm{Mes} / \mathrm{glom})$ is the measure that best correlates with all functional parameters, including GFR. A crucial role for mesangial expansion in the early stages of diabetic nephropathy has been supported by the study of sequential renal biopsies performed 5 years apart in a group of long term IDDM patients, some of whom progressed from normo- to microalbuminuria or from micro- to overt proteinuria. In these patients the increase in urinary albumin excretion rate (UAER) was closely associated with increasing $\mathrm{Vv}(\mathrm{Mes} / \mathrm{glom})$, while GBM width, $\mathrm{Vv}$ (Int/cortex) and the number of globally sclerotic glomeruli did not change [1]. Thus, in the early stages of diabetic nephropathy, it seems that interstitial expansion does not have a primary role in pathogenesis; however in more advanced stages of the disease this lesion may be more important in terms of progression. Glycaemic control $\left(\mathrm{HbA}_{1 \mathrm{c}}\right)$ is strongly related to GBM and TBM width and less precisely to $\mathrm{Vv}(\mathrm{Mes} /$ glom) and $\mathrm{Vv}$ (Int/cortex). Also GBM and TBM thickening occur early in the course of IDDM and are highly correlated. These TBM changes argue against glomerular haemodynamic abnormalities driving the genesis of the earlier lesions of diabetic nephropathy.

M icroalbuminuria: Increased UAER is often the earliest functional manifestation of diabetic nephropathy, and is a marker of increased risk for overt nephropathy. However, recent studies suggest that about $25 \%$ of IDDM patients, still normoalbuminuric (NA) after 15 years of IDDM, progress to microalbuminuria (MA) or to proteinuria over the subsequent 10 years, while about $25 \%$ of MA either remain MA or revert to NA over the same time. The structural studies in NA and MA patients with long term IDDM are in keeping with these clinical observations [2]. In fact, measures of GBM width and $\mathrm{Vv}(\mathrm{Mes} / \mathrm{glom})$ are often increased in NA patients 
(UAER $<15 \mu \mathrm{g} / \mathrm{min}$ ) compared to normal control subjects, although patients may have glomerular structural parameters in the normal range. Patients with low levels of MA (15-30 $\mu \mathrm{g} / \mathrm{min})$ have structural measures similar to NA patients, whereas in patients with UAER values exceeding $30 \mu \mathrm{g} / \mathrm{min}(30-150 \mu \mathrm{g} /$ min) diabetic glomerulopathy is significantly more advanced. However, substantial variability exists within the groups, with overlap among NA, low level MA and MA patients [2]. Nevertheless, these studies demonstrate that MA defines a group of IDDM patients with well established diabetic glomerular lesions.

Thus, since patients with early functional abnormalities (MA) tend to have quite advanced renal lesions, in order to develop preventative strategies it may be important to focus on earlier stages of the disease, when UAER is still within the normal range.

Early lesions of diabetic nephropathy: In an attempt to identify risk factors and early markers for diabetic nephropathy, the International Diabetic Nephropathy Study Group has enrolled 252 IDDM patients with normal renal function. This longitudinal study involves sequential kidney biopsies (5 years apart) and repeated measurements of metabolic control, renal function and other parameters. Although the study is in progress, analysis of the baseline observations demonstrates that, despite UAER less than $20 \mu \mathrm{g} / \mathrm{min}$, these patients have early diabetic glomerulopathy, with increased GBM width and $\mathrm{Vv}(\mathrm{Mes} /$ glom). Both age and IDDM duration, but not $\mathrm{HbA}_{1 \mathrm{c}}$, blood pressure or GFR predicted $\mathrm{Vv}(\mathrm{Mes} /$ glom) at baseline. GBM width was significantly related to $\mathrm{HbA}_{1 \mathrm{c}}$ levels and IDDM duration only. Interestingly there were significant between-centre differences in the patterns of glomerular lesions; patients from Minneapolis and Montreal had higher Vv(Mes/ glom) than patients from Paris, who had larger GBM width. Thus, genetic and/or environmental factors may influence the expression of the patterns of early glomerular lesions in IDDM.

Is diabetic nephropathy risk inherited? It is known that IDDM sibling pairs are highly concordant for diabetic nephropathy risk. Kidney biopsy studies found a strong familial concordance for diabetic glomerular lesions, especially $\mathrm{Vv}(\mathrm{Mes} / \mathrm{glom})$, which persisted after factoring for IDDM duration. Furthermore there was concordance in the pattern of glomerular lesions among the sibling pairs; thus if one sibling exhibited a greater increase in GBM width relative to mesangial expansion, the other sibling was highly likely to display the same pattern. This concordance was not due to similar glycaemic control or blood pressure and is consistent with a strong familial, possibly genetic, predisposition to or protection from diabetic nephropathy [1].
Cellular studies: Cultured skin fibroblasts from IDDM are easily available and probably reflect intrinsic and genetic characteristics rather than environmental variables. Several studies have shown significant increases in both the proliferation rate and $\mathrm{Na}^{+} / \mathrm{H}^{+}$antiporter activity in fibroblasts obtained from patients with, compared to those without, nephropathy. In support of a familial/genetic predisposition to nephropathy, preliminary data demonstrate that cultured skin fibroblasts from IDDM siblings exhibit remarkable concordance in their $\mathrm{Na}^{+} / \mathrm{H}^{+}$antiporter activity. Thus studies of cultured skin fibroblasts from IDDM patients undergoing kidney biopsies may help to understand the basic processes leading to renal injury (extracellular matrix accumulation) in diabetes, as well as the identification, perhaps through the detection of some cellular abnormality, of patients who are developing serious renal lesions in the absence of clinical findings of nephropathy.

For example, preliminary studies of cultured skin fibroblasts from patients with rapid compared to slow development of diabetic glomerulopathy have increased mRNA for several extracellular matrix constituents and regulators.

These studies provide hope that cellular or genetic studies might detect IDDM patients at high risk for nephropathy before advanced lesions have developed.

\section{Residual islet cell function and progression of complications}

Animal work has shown conclusively that structural changes in the kidney can be reversed by islet transplantation. Whole organ pancreas transplantation in IDDM patients with varying degrees of glomerulopathy showed no significant reversal of lesions after 5 years, but, intriguingly, significant reductions in glomerular basement membrane width were observed in a small subset of this cohort after 10 years. Thus preservation or restitution of beta-cell function seems to confer benefit in terms of the development of microvascular complications [1].

In the Diabetes Control and Complications Trial (DCCT), patients underwent yearly Sustacal tests for the presence of stimulated C-peptide. 303 patients with an initial response of over $0.20-0.50 \mathrm{pmol} / \mathrm{ml}$ at 90 min were defined as "responders", and had a lower $\mathrm{HbA}_{1 \mathrm{c}}$ for the first 4 years of the study than the respective "non-responders" in the intensive and conventional treatment groups to which they had been randomised at baseline. Moreover, the intensively treated subjects retained a significantly higher C-peptide responsiveness compared to the conventional group over the same period, although by 6 years this difference was lost, probably because of fewer patients. Although responders on intensive compared 
to conventional therapy had a higher rate of hypoglycaemia ( $\sim 6 / 100$ patient years of treatment), this was significantly lower than non-responders in the intensive arm. In addition, responders in both treatment groups had lower rates of development of retinopathy and nephropathy. Thus these patients (responders) represented the only circumstance whereby intensive insulin therapy not only lowered rates of complications but also had a lower incidence of hypoglycaemia [3].

Responders were older at onset of IDDM, but no information was available on insulin type and dose, although there was speculation that overnight or medium/longer acting insulin requirements may be less. Whether intensive therapy preserves islets or just enables the decreasing residual number (as a result of continuing immunological attack) to function better is speculative. Could C-peptide response be used to identify those at a lower risk of both acute (e.g. hypoglycaemia) and chronic complications? What is it that confers benefit? Does C-peptide itself have a therapeutic role as presented at this conference in a different session? At first sight this seems unlikely as NIDDM patients who have preserved (albeit inadequate) beta-cell function still develop complications.

Is there a threshold of glycaemia for nephropathy development? This highly controversial area was also discussed in some detail. The most recent paper from the DCCT concluded that there was no threshold effect, but the baseline data could also be construed as indicating a much greater risk of progression of retinopathy and development of nephropathy at $\mathrm{HbA}_{1 \mathrm{c}}$ levels over 8-9\%. Furthermore, an alternative interpretation of the outcome data much more closely resembles the conclusions of Krolewski et al. with a greatly increasing risk of development of nephropathy once $\mathrm{HbA}_{1 \mathrm{c}}$ exceeds $8 \%$ [4]. Recent analysis of later cohorts of patients from the Joslin Clinic both confirm and strengthen the original findings.

Either way, the recent DCCT publication confirms that there was a continuing benefit of reduced glycaemia with a continuous risk of hyperglycaemia, but that every reduction in $\mathrm{HbA}_{1 \mathrm{c}}$ helped, perhaps more so at higher values.

This crucial issue of threshold needs resolving as the rate of severe hypoglycaemia in the DCCT increased dramatically once $\mathrm{HbA}_{1 \mathrm{c}}$ levels dropped to less than $8 \%$.

\section{Prevention of progression of diabetic nephropathy}

This topic was addressed by approaching the problem from the viewpoints of end-stage renal disease (ESRD) and microalbuminuria, respectively. Because there was a degree of overlap between them, the whole area will be reported as one, under the sub-headings of primary (normo to microalbuminuria); secondary (microalbuminuria to clinical nephropathy); and tertiary (clinical proteinuria to ESRD) prevention, respectively.

For the most part, the reported studies have concentrated on surrogate end-points such as changes in UAER and thus extrapolation to positive or negative benefit in terms of mortality, rate of decline of GFR, or development of ESRD must be guarded. Nonetheless, diabetes remains the commonest single cause of ESRD requiring renal replacement therapy in the United States at $33.8 \%$, and the second commonest in Europe at $17 \%$. Diabetic patients do much less well on dialysis in terms of morbidity and mortality; thus, preventative strategies offer potentially large benefits for both patients and health care providers. Finally, most published work relates to IDDM, few data are available for NIDDM which remains a very important but relatively understudied area.

\section{P rimary prevention}

G lycaemic control: The DCCT is the largest primary prevention prospective study to have been completed with sufficient statistical power to demonstrate a positive impact of improved glycaemic control on microvascular complications. Although primarily a study of retinopathy, the results also showed for the first time that microalbuminuria could be prevented by good metabolic control with an almost $40 \%$ reduction in the intensively treated groups. Despite this, however, $16 \%$ in the primary prevention and $26 \%$ in the secondary prevention cohort developed microalbuminuria for reasons that are not yet clear, but it was shown in this meeting that $\mathrm{HbA}_{1 \mathrm{c}}$ at baseline determined later outcome to a certain extent. Thus, patients may have been "programmed" onto a track leading to complications prior to entry into the study [5].

A much smaller study in Japanese NIDDM patients with a similar protocol to the DCCT also showed a significant reduction in those developing microalbuminuria in the intensively treated group [6]. These patients were all less than 50 years of age and not particularly overweight so the translation of these results to Europid patients may not be appropriate. The UK Prospective Diabetes Study is collecting data on such subjects and is due to report very soon.

Two studies of the effect of glycaemic control on glomerular pathology have been reported. One from Norway showed that 22-36 months of intensive therapy reduced the rate of glomerular basement membrane thickening in native kidneys compared to those on conventional treatment. In renal allografts, mesangial changes from baseline were reduced in 
patients after 5 years' intensive insulin therapy compared to those on standard regimens [7].

Thus, improved glycaemia prevents both structural and functional expressions of diabetic kidney disease. Some discussion about the cumulative incidence rates of nephropathy revealed that the previously observed decreases in cohorts of patients diagnosed with diabetes in the $1960 \mathrm{~s}$ and $1970 \mathrm{~s}$ are not continuing and that the total is plateauing at approximately $25 \%$ at 25 years' duration in the Joslin and Danish series. It was speculated that the previously noted reductions were due to the decrease in other potential insults to the glomerulus such as post-streptococcal nephritis, although no such heterogeneity in pathology was reported in historical biopsy series. It remains much more likely that the reduction was due to improvement in overall care, including glycaemia and perhaps blood pressure.

\section{Secondary prevention}

G lycaemic control: The results from the DCCT in the 78 patients who had microalbuminuria at baseline are much less clear. It is true that relatively more patients with higher levels of microalbuminuria ( $>100 \mathrm{mg} /$ day) were randomised to the intensive group, but the lack of an overall impact on progression to clinical nephropathy was surprising, particularly in the light of the Steno studies and a meta-analysis performed prior to the DCCT report suggesting a positive benefit of improved glycaemia [5]. A more recently reported study from the United Kingdom, which also failed to demonstrate an impact of improved glycaemia, might suggest that microalbuminuria is already a "stage too far" for any benefit to accrue, although this latter study failed to achieve prolonged glycaemic separation in the treatment groups for more than 3 years [7].

The previously mentioned study in NIDDM Japanese patients remains the only evidence so far of benefit of glycaemic control in NIDDM [6].

A nti-hypertensive therapy: IDDM patients with microalbuminuria are usually normotensive by conventional criteria, although sensitive techniques such as $24 \mathrm{~h}$ ambulatory blood pressure monitoring have demonstrated significantly higher readings in some, but not all, reported series. Mathiesen observed that approximately $45 \%$ of microalbuminuric patients will be on anti-hypertensive therapy within 10 years of developing increased UAER.

A ngiotensin converting enzyme inhibitors: The Microalbuminuria Collaborative Study Group combined the results from two broadly similar studies from Europe and the United States which looked at the effects of captopril compared to placebo in 111 and 114 normotensive, microalbuminuric IDDM patients over a 2 -year period. A $62.9 \%$ reduction in risk for progression to clinical nephropathy was seen in those patients randomised to captopril, and in the eight patients on angiotensin converting enzyme inhibitors (ACEI) who progressed the determining factors were blood pressure and $\mathrm{HbA}_{1 \mathrm{c}}$ at baseline. Moreover, mean blood pressure in the 33 who progressed was significantly higher at $94 \mathrm{mmHg}$ compared to $87 \mathrm{mmHg}$ in the 192 who did not, irrespective of treatment. Mean blood pressure was also lower in the captopril treated group as a whole, although the final statistical analysis allowed for this [8].

In NIDDM two studies have shown a similar reduction in risk of around two thirds in Israeli and Japanese patients. Both of these studies were in relatively young subjects however, and the translation of the results to older, more obese and clinically complicated Europid patients may be difficult [7]. Nonetheless, the similarity in results has suggested a broadly similar disease pattern in the two types of diabetic patient to some workers, who have gone on to recommend ACEI for microalbuminuria even before blood pressure rises into the conventionally defined hypertensive range [9].

However, some caution needs to be shown because ACEI reduce proteinuria in non-diabetic renal disease, and renal structure in NIDDM is more heterogeneous than in IDDM diabetes. Moreover, withdrawal of treatment in diabetic patients results in an increase in UAER to levels at or above those at initiation of treatment and there is an almost total lack of evidence of long term benefit in terms of more concrete end-points. It is possible that ACEI may mask and not prevent the nephropathic process. Ongoing studies of the impact of various anti-hypertensive therapies on renal structure and function are due to report soon and may shed some light on this important question.

\section{Tertiary prevention}

G lycaemic control: Conventional wisdom has it that improved glycaemic control cannot prevent or slow down progression to ESRD although the studies on which this opinion is based are small and very underpowered from a statistical point of view. Indeed, they were for the most part conducted before rigorous control of blood pressure was established practice and, because of this, some have suggested that intensive insulin therapy may have an impact once adequate blood pressure levels have been achieved. It must also be remembered that many clinically nephropathic patients will have retinopathy at a stage where glycaemia may have an impact on progression, so the best possible control is still recommended. 
A nti-hypertensive therapy: The now historical studies of Mogensen and Parving have established that careful control of blood pressure can significantly reduce the rate of loss of GFR in IDDM patients. These studies used non-ACEI therapy for the most part.

A ngiotensin converting enzyme inhibitors: The Collaborative Study Group showed that 4 years of captopril treatment significantly reduced by approximately $50 \%$ the numbers of IDDM patients with nephropathy and hypertension (controlled on other drugs except calcium channel blockers, and who had moderate renal impairment with a baseline serum creatinine of $>133 \mu \mathrm{mol} / 1$ ), progressing to ESRD and/or death, compared to placebo [10]. Again there was a slightly lower blood pressure in those on active treatment but the final analysis made allowance for this. It is also true that the captopril treated patients had a very similar slope of cumulative incidence of study end-points, but this appeared to be shifted to the right by a time factor of around 2 years. Thus the impact may have been a delay rather than a prevention. Nonetheless, this is the first time that any intervention in diabetic patients has been shown to have a significant impact on mortality and morbidity. Since diabetic patients do so less well on dialysis any delay represents significant benefit.

There is now debate as to whether a combination of differing anti-hypertensive treatments may give added benefit. For example, the cardioprotective effects of beta blockers, or certain calcium channel blockers such as verapamil, plus an ACEI may be logical but as yet largely untested combinations.

In NIDDM no large studies have been performed but the limited data available suggest that ACEI reduce proteinuria to a similar degree as in IDDM. However, there needs to be caution with the initiation of these drugs in elderly patients who will have a higher prevalence of macrovascular disease and possibly undiagnosed renal artery stenosis, and it is important to remember that atherosclerosis is a developing process and de novo renal artery disease may develop in a patient already established on ACEI. Several large studies of angiotensin II receptor antagonists in nephropathic NIDDM patients are ongoing and due to report in 3-4 years.

L ow protein diet: A recent meta-analysis of five studies of protein restriction to $0.5-0.85 \mathrm{~g} / \mathrm{kg}$ body weight in a total of 108 patients suggested a positive benefit with a relative risk of 0.56 [11]. However, in at least one study the response was very heterogeneous and long term compliance was difficult. A dietary survey of protein intake across Europe showed a range from $1.25-2.0 \mathrm{~g} / \mathrm{kg}$ body weight with perhaps the highest intake in southern European cities. Interestingly, Helsinki was at the lower end of the range and has possibly the highest incidence of diabetic
ESRD in IDDM. One study combining intensive insulin therapy, anti-hypertensive treatment including ACEI and a low protein diet actually showed an increase in GFR although the general applicability of this regimen to routine diabetes care is doubtful.

O ther potential interventions: Lipid lowering drugs have been proposed but remain largely untested long term in nephropathy.

There is a higher percentage of smokers among patients with nephropathy but the impact of stopping has not been studied in detail.

Aminoguanidine is currently undergoing trials in NIDDM and IDDM patients with ESRD.

Aldose reductase inhibitors may reduce GFR in hyperfiltering patients with normal UAER but their effect in established nephropathy is unknown.

More experimental agents such as protein kinase $\mathrm{C}$ and growth factor inhibitors are yet to be evaluated in man.

\section{Prognosis}

Despite all the uncertainty there have been major developments in our understanding of the pathophysiology and ability to treat diabetic nephropathy, and these advances have been one of the success stories in diabetes care of the last 10-20 years. Cumulative mortality that approached $100 \%$ only 30 years ago is now around one fifth that figure due to a combination of better diabetic control, lower blood pressure and a greater access to renal replacement therapy programmes. While it is probably optimistic to talk of complete prevention, it is certainly possible to ameliorate this serious diabetic complication, and there is the real prospect of considerable reduction in numbers of patients entering ESRD in the foreseeable future.

\section{Future directions and recommendations}

- Natural history studies. Continue to gather information on how diabetic nephropathy develops, what structural changes are associated with clinical progression and what clinical variables influence clinical progression. Studies could provide essential information for the design of early intervention trials initiated before serious renal damage has developed. Develop new endpoints that are highly relevant to clinical outcome.

- Familial studies. Confirm and enlarge family studies of diabetic nephropathy in order to understand the nature of concordance in nephropathy risk among IDDM sibling pairs. Generate material necessary to perform careful and appropriate genetic analyses in order to gain insights into pathogenetic mechanisms. 
- G enetic studies. Increase the scientific material obtained from current human studies for storage until newer technologies are available and questions raised. This should include genetic (DNA, RNA) material from blood or tissues, cultured cells, and kidney biopsy material. Networking of scientists is highly recommended in order to optimize the use of precious human scientific resources for present and future studies.

- Predictors of nephropathy. Find markers which identify patients at high risk for diabetic nephropathy before the lesions are advanced and irreversible, because lesions are already established by the time microalbuminuria develops.

- Glycaemic control. Confirm the presence or absence of a glycaemic threshold for nephropathy in the light of the rapid increase in severe hypoglycaemia when $\mathrm{HbA}_{1 \mathrm{c}}$ is less than $8 \%$.

- UAER. Understand whether agents which prevent increasing UAER in microalbuminuric patients are slowing the progression of glomerulopathy and reducing the risk of ESRD or are merely masking the functional expression of nephropathy.

- Combined therapies. Determine the additive or synergistic potential of combined therapeutic strategies (e.g. glycaemic control and ACEI; multiple antihypertensive agents).

- N ew agents. Discover treatment approaches using recently established agents (e.g. lipid lowering drugs) and develop new strategies based on emerging pathogenetic insights (e.g. growth factor blockers).

- NIDDM. Establish applicability of treatment advances in IDDM to the more complex problem of renal disease in NIDDM.

\section{References}

1. Mauer M, Mogensen CE, Friedman E (1996) Diabetic nephropathy. In: Schrier RW, Gottschalk CW (eds) Diseases of the kidney, 6th edn. Little Brown \& Co. Vol 3, pp 20192062

2. Fioretto P, Steffes MW, Mauer M (1994) Glomerular structure in nonproteinuric IDDM patients with various levels of albuminuria. Diabetes 43: 1358-1364

3. Steffes MW, Tamborlane W, Becker D, Palmer J, Cleary P for the DCCT Research Group (1996) The effect of intensive diabetes treatment on residual beta cell function in the Diabetes Control and Complications Trial (DCCT). Diabetes 46[Suppl 2]:18A (Abstract)

4. Krolewski AS, Laffel LMB, Krolewski M, Quinn M, Warram JH (1995) Glycosylated hemoglobin and the risk of microalbuminuria in patients with insulin-dependent diabetes mellitus. N Engl J Med 332: 1251-1255

5. The Diabetes Control and Complications (DCCT) Research Group (1995) Effect of intensive therapy on the development and progression of diabetic nephropathy in the Diabetes Control and Complications Trial. Kidney Int 47: 1703-1720

6. Ohkubo Y, Kishikawa H, Araki E et al. (1995) Intensive insulin therapy prevents the progression of diabetic complications in Japanese patients with non-insulin-dependent diabetes mellitus: a randomized prospective 6 year study. Diabetes Res Clin Pract 28: 103-117

7. Bilous RW (1996) Early diagnosis of diabetic nephropathy. Diabet Metab Rev 12: 243-253

8. Microalbuminuria Captopril Study Group (1996) Captopril reduces the risk of nephropathy in insulin-dependent diabetic patients with microalbuminuria. Diabetologia 39: 587-593

9. Mogensen CE, Keane WF, Bennett PH et al. (1995) Prevention of diabetic renal disease with special reference to microalbuminuria. Lancet 346: 1080-1084

10. Lewis EJ, Hunsicker LG, Bain RP, Rohde R for the Collaborative Study Group (1993) The effect of angiotensin-converting-enzyme inhibition on diabetic nephropathy. N Engl J Med 329: 1456-1462

11. Pedrini MT, Levey AS, Lau J, Chalmers TC, Wang PH (1996) The effect of dietary protein restriction on the progression of diabetic and non-diabetic renal diseases: a meta analysis. Ann Int Med 124: 627-632 